\title{
Looking for Medications to Support the Treatment of Acute Decompensated Heart Failure
}

\author{
Robert Zymliński $^{a} \quad$ Janina Stepinska ${ }^{b}$ \\ ${ }^{\mathrm{a}}$ Department of Heart Diseases, Wroclaw Medical University, Wroclaw, Poland; ${ }^{\mathrm{b}}$ Department of Intensive Cardiac \\ Therapy, National Institute of Cardiology, Warsaw, Poland
}

Acute heart failure (AHF) is still one of the leading causes of hospital admissions, with a high post-discharge mortality and rehospitalization risk. Unlike congestive $\mathrm{HF}$, in AHF there is no established therapy available that improves clinical outcome [1]. One of the reasons for this phenomenon is its complex and not fully understood pathophysiology.

Regardless of phenotypic diversity, congestion is the main clinical sign, and is observed in nearly $95 \%$ of all AHF patients. A variety of hemodynamic, neurohormonal abnormalities provoked by the congestion (volume overload) may ultimately lead to impaired systemic perfusion with further secondary organ dysfunction and, in many cases, irreversible damage and chronic failure. Hence, baseline clinical assessment should determine whether volume overload or volume redistribution is the predominating mechanism of congestion $[2,3]$. In the case of volume overload, loop diuretics are the mainstay decongestive therapy, and approximately $90 \%$ of all AHF patients receive loop diuretics. However, despite early use, loop diuretic strategy may be unsuccessful, and many patients are discharged with residual congestion, which is related to poor outcome [4].

One of the disadvantages of the loop diuretic treatment, which may be the reason for diuretic resistance, is a volume depletion caused by too fast fluid removal from the intravascular compartment, with an inadequate refill- ing rate from the extravascular compartment. Thus, there is a great need to optimize volume removal, while preventing uncontrolled hypovolemia during decongestion to avoid the consequence of hypotension [5]. In addition, renal failure and worsening HF associated with renal function deterioration during treatment is common and is related to an impaired outcome, especially when diuretic response is poor [6]. Therefore, nowadays, we are looking for safe and effective decongestion procedures.

As effective decongestion is still the gold standard of AHF treatment, it is not surprising that new modes of therapy are under investigation. Herein, the newly proposed stepped guided pharmacological treatment is focused on achieving complete decongestion. Moreover, natriuresis has now become a strongly postulated marker of effective volume removal [7, 8].

So far, body fluid balance was understood through the systemic interactions of sodium $(\mathrm{Na})$, potassium, and extra/intravascular and intracellular water, with volume regulation as a process involving the various sensory and neurohumoral mechanisms [8]. Hence, new guided diuretic therapy using even more advanced support has been tested to provide the clinician with greater control of fluid loss during diuretic administration $[6,8]$.

Recently published studies suggest that in HF patients, chloride $(\mathrm{Cl})$, an established key electrolyte for the activation of the renin-angiotensin-aldosterone system, may

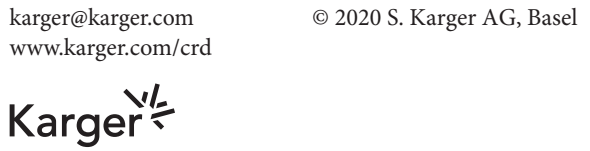

Janina Stepinska

Department of Intensive Cardiac Therapy

National Institute of Cardiology, Alpejska 42

PL-04628 Warsaw (Poland)

janina@stepinska.pl.pl 
play an important role in the regulation of body fluid distribution. It is postulated that the significance of the decrease of serum $\mathrm{Cl}$ concentration for recognizing signs of congestion recovery declines as well as its association with diuretic resistance under treatment [9].

In a previously published paper, Kataoka [9] has established the role played by $\mathrm{Cl}$ in the renin-angiotensin-aldosterone system. Herein, the so-called unifying hypothesis of the "chloride theory" for HF pathophysiology states that changes in the serum $\mathrm{Cl}$ concentration are the primary determinant of changes in plasma volume and in the renin-angiotensin-aldosterone system.

According to a well-known physiological mechanism, adequate kidney function is mainly due to the ability of the renal circulation system to preserve GFR through selfregulation, and to adjust the kidney blood-flowing response to perfusion fluctuations $[7,10]$. In the case of a decrease in mean arterial blood pressure, the essential volume is redistributed to preserve adequate renal blood flow. Concomitantly, to protect the glomerulus from hyperfiltration, an additional mechanism called "tubuloglomerular feedback," maintains $\mathrm{Cl}^{-}$load via Henle's loop at a constant level, with a further increase in GFR and the enhanced delivery of $\mathrm{Cl}^{-}$to macula densa cells. Furthermore, it activates adenosine release with further vasoconstriction of the afferent arteriole and restoration of GFR. Additionally, empowered by $\mathrm{Cl}$, the reabsorption of the filtered $\mathrm{Na}$ from the urinary tubules into the extracellular body spaces maintains arterial pressure. In the complex mode of diuretic treatment strategy, an old well-known drug, acetazolamide, seems to be attracting attention. This is a carbonic anhydrase inhibitor that blocks sodium bicarbonate reabsorption in the proximal tubules [10].

The targeting of $\mathrm{Na}+$ reabsorption has several potential benefits in AHF. Indeed, blocking $\mathrm{NaCl}$ reabsorption proximally will provide more flow to distal parts of the nephron, including macula densa cells - this being the mechanism of renin concentration decrease and improvement of loop diuretic efficacy. In addition, acetazolamide enhances thiazide-type diuretic efficacy, as it potently downregulates pendrin expression in the distal nephron $[7,10,11]$.

Beyond the aforementioned, additional Cl-regaining activity brought about due to the use of acetazolamide might be effective for patients with refractory HF, as this drug has a beneficial intrinsic renal vasodilatory effect through stimulating nitric oxide-dependent vasodilatation. Moreover, it may play a crucial role in nephron protection in the case of hypoperfusion. Thus, despite some limitations, as well as the poor diuretic and natriuretic

Medications to Support the Treatment of Acute Heart Failure capacity of acetazolamide, such incorporated complex therapy may increase diuretic efficacy in combinational therapy with loop diuretics [10].

In this issue of Cardiology, Kataoka [12] extended his retrospective, single-center, observational study evaluating the different effects of acetazolamide (Diamox) and conventional diuretics on the changes in plasma volume, renal function, and serum electrolytes in HF. The analysis was based on the data of 13 patients treated with acetazolamide (Group A), and 13 patients with conventional diuretics (Group B). Accordingly, the body weight reduction and the residual HF signs were not different between both groups $(2.23 \pm 1.11$ vs. $-2.22 \pm 1.06 \mathrm{~kg}, p=$ 0.97 , and $0.31 \pm 0.48$ vs. $0.23 \pm 0.44, p=0.67$, respectively). However, the serum $\mathrm{Cl}$ concentration was significantly increased in the acetazolamide group (Group A) in comparison to the standard loop diuretic group (Group B) $(5.31 \pm 4.91$ vs. $-4.54 \pm 4.68 \mathrm{mEq} / \mathrm{L}, p<0.0001$, respectively). Moreover, plasma volume $(0.63 \pm 13.1$ vs. $-12.1 \pm 10.5 \%, p<0.01)$ and renal function determined by changes in serum creatinine $(0.048 \pm 0.12$ vs. $0.21 \pm$ $0.24 \mathrm{mg} / \mathrm{dL}, p<0.047)$ were preserved in Group A in comparison to Group B. Furthermore, despite the observed equivalent body weight reduction and resolution of HF-related signs in both diuretic treatment regimens, acetazolamide use was related with preserved plasma volume and renal function, in comparison to conventional diuretics [12].

Are such results important? In our opinion, these are the most interesting findings. The target of decongestive therapy is to reduce the volume overload without depletion of plasma volume and kidney deterioration. When compared with conventional $\mathrm{Cl}$-depleting diuretic treatment, the acetazolamide $\mathrm{Cl}$-regaining diuretic treatment might be the one desired, safe, and effective treatment protocol. This is because it results in adequate $\mathrm{Cl}$ concentration and the related preservation of plasma volume and renal function. In addition, while it is known that an increase or supply of $\mathrm{Cl}$ in the plasma may sometimes induce residual cardiac volume overload, the magnitude of the serum BNP concentration induced by diuretic treatment with acetazolamide was smaller than that induced by conventional diuretics [10].

Regardless of the significant limitations mentioned by the author, the obtained results are very interesting and promising. In current practice, one of the undesirable effects associated with decongestion is uncontrolled intravascular volume depletion $[6,7,10]$. This stimulates central and peripheral volume and Na-retaining mechanisms with further urine output decrease. Decongestion should 
reduce the volume overload without depletion of plasma volume. Hence, a positive and independent association between vascular volume and the serum $\mathrm{Cl}$ concentration after adequate decongestive therapy in AHF is a promising novel finding.

We strongly agree with the author that, to date, the targeting level of serum $\mathrm{Cl}$ concentration is still not fully understood. Furthermore, the role of $\mathrm{Cl}$ has been ignored because of lack of awareness of its role in HF. However, recently presented studies support the need to undertake a profound revision of the current role of this electrolyte in the HF syndrome [9]. New well-designed studies scheduled to explore the clinical significance of this electrolyte in the HF syndrome are needed. Therefore, it seems to be desirable to modulate the serum $\mathrm{Cl}$ concentration so as to improve and make safer diuretic efficacy. Finally, despite the use of loop diuretics since the 1960s, systemic fluid balance and plasma volume control in AHF is not fully understood $[10,11]$.
According to the pilot study presented by Kataoka, application of an acetazolamide or a vasopressin receptor antagonist may change the present paradigm of decongestive therapy - that is, that it is not only fluid and $\mathrm{Na}$ removal. Thus, in using a well-established treatment, we may improve hemodynamics and neurohormonal status in HF through the favorable action of these agents on the reninangiotensin-aldosterone system. This will result in possible beneficial changes in plasma volume, hemodynamics, and neurohormonal status in HF pathophysiology.

Thereby, it should be noted that complex treatment comprising different diuretic classes and their various activities might become an attractive therapy for HF. Therefore, we may agree with Kataoka that "in HF status accompanied by hypochloremia, changing the loop diuretic-based decongestion treatment by using acetazolamide or a vasopressin receptor antagonist so as to achieve effective decongestion chloride-regaining diuretic therapy may result in better after effects."

\section{References}

1 AlFaleh H, Elasfar AA, Ullah A, AlHabib KF, Hersi A, Mimish L, et al. Worsening heart failure in "real-world" clinical practice: predictors and prognostic impact. Eur J Heart Fail. 2017 Aug;19(8):987-95.

2 Ponikowski P, Voors AA, Anker SD, Bueno H, Cleland JG, Coats AJ, et al.; Authors/Task Force Members; Document Reviewers. 2016 ESC Guidelines for the diagnosis and treatment of acute and chronic heart failure: the Task Force for the diagnosis and treatment of acute and chronic heart failure of the European Society of Cardiology (ESC). Developed with the special contribution of the Heart Failure Association (HFA) of the ESC. Eur J Heart Fail. 2016 Aug;18(8):891-975.

3 Rubio-Gracia J, Demissei BG, Ter Maaten JM, Cleland JG, O'Connor CM, Metra M, et al. Prevalence, predictors and clinical outcome of residual congestion in acute decompensated heart failure. Int J Cardiol. 2018 May;258: 185-91.
4 Valente MA, Voors AA, Damman K, Van Veldhuisen DJ, Massie BM, O'Connor CM, et al. Diuretic response in acute heart failure: clinical characteristics and prognostic significance. Eur Heart J. 2014 May;35(19):1284-93.

5 Martens P, Nijst P, Mullens W. Current approach to decongestive therapy in acute heart failure. Curr Heart Fail Rep. 2015;12(6):36778.

6 Testani JM, Brisco MA, Chen J, McCauley BD, Parikh CR, Tang WH. Timing of hemoconcentration during treatment of acute decompensated heart failure and subsequent survival: importance of sustained decongestion. J Am Coll Cardiol. 2013 Aug;62(6):51624.

7 Mullens W, Damman K, Harjola VP, Mebazaa A, Brunner-La Rocca HP, et al. The use of diuretics in heart failure with congestion - a position statement from the Heart Failure Association of the European Society of Cardiology. Eur J Heart Fail. 2019 Feb;21(2):137-55.
8 Biegus J, Zymlinski R, Siwolowski P, Testani J, Szachniewicz J, Tycińska A, et al. Controlled decongestion by Reprieve therapy in acute heart failure: results of the TARGET- 1 and TARGET-2 studies. Eur J Heart Fail. 2019 Sep;21(9):1079-87.

9 Kataoka H. The "chloride theory", a unifying hypothesis for renal handling and body fluid distribution in heart failure pathophysiology. Med Hypotheses. 2017 Jul;104:170-3.

10 Verbrugge FH, Martens P, Ameloot K, Haemels V, Penders J, Dupont M, et al. Acetazolamide to increase natriuresis in congestive heart failure at high risk for diuretic resistance. Eur J Heart Fail. 2019 Nov;21(11):1415-22.

11 Imiela T, Budaj A. Acetazolamide as add-on diuretic therapy in exacerbations of chronić heart failure: a pilot study. Clin Drug Investig. 2017 Dec;37(12):1175-81.

12 Kataoka H. Comparison of changes in plasma volume and renal function between acetazolamide versus conventional diuretics: understanding their mechanical differences according to the "chloride theory". Cardiology. 2019. doi: 10.1159/000504533. Epub 2019 Dec. 19. 